\title{
Emerging fundamental roles for non-coding RNA species in toxicology
}

Emma.L. Taylor and Timothy.W. Gant

Medical Research Council Toxicology Unit, University of Leicester, Systems Toxicology

Group, Lancaster Road, Leicester, LE1 9HN, UK

Corresponding author: Emma.L. Taylor (elt14@le.ac.uk)

Keywords: microRNA; toxicology; mRNA translation; non-coding RNA

\begin{abstract}
microRNAs (miRNAs) are a large family of small regulatory RNA molecules found in all multicellular organisms. Since their discovery in 2001, there has been impressive progress in miRNA research, and a great deal is now known about the biosynthesis of mRNAs and their regulatory role in translation. It is becoming increasingly clear that miRNAs have fundamental roles to play in cellular responses to xenobiotic stress, the development of pathophysiological changes and other toxicological phenomenon such as susceptibility and resistance. Furthermore, the expression of miRNAs, like many of the genes important in toxicology, can be regulated by xenobiotics and DNA methylation. In this article we review the present understanding of the miRNA field with particular reference to toxicology. We also give an insight into our current projects within this exciting area and highlight some of the new challenges that now face miRNA research.
\end{abstract}


What are miRNAs?

microRNAs (miRNAs) are short (18-26 nucleotide) RNA molecules that play an important role in translational regulation. They are endogenously produced and their biosynthesis involves a number of processing steps (Fig. 1). Long primary miRNA (primiRNA) molecules are transcribed from non-coding polycistronic regions of the genome under the control of RNA polymerase II (Pol II) promoters (Zhou et al., 2007). These pri-miRNA transcripts can exceed $1 \mathrm{~kb}$ and may give rise to a number of different miRNAs (Du and Zamore, 2005). The nuclear ribonuclease III (RNase III) Drosha, together with its double-stranded RNA-binding domain (dsRBD) protein partner DGCR8, cleave the pri-miRNA to yield a 70-100 nt stem loop precursor miRNA (pre-miRNA) molecule (Du and Zamore, 2005; Kosik, 2006). The pre-miRNA is then exported from the nucleus into the cytosol by Exportin5, and is cleaved by a second RNase III Dicer and its dsRBD protein partner TRBP to produce a mature miRNA duplex (Du and Zamore, 2005; Kosik, 2006). The duplex unwinds and the guide miRNA strand is incorporated into the RNA-induced silencing complex (RISC), where it binds to the 3'UTR of, and silences, its target miRNA (Du and Zamore, 2005; Kosik, 2006). This silencing can result in degradation or repressed translation of the target mRNA, and probably depends on the degree of complementarity between the miRNA and its target (Kosik, 2006; Meister, 2007). The majority of miRNAs in animals exhibit only partial complementarity to their mRNA targets and so are thought to function predominantly by repressing target translation, most likely through the inhibition of translational initiation (Meister, 2007). The exact mechanism of miRNA-mediated translation repression, however, is beyond the scope of this article and is reviewed in Meister, 2007 and Rana, 2007. 
microRNAs and toxicology

It is now clear that miRNAs have a fundamental role in normal development and in disease pathology, particularly cancer. The fact that miRNA transcription involves pol II promoters, which often contain toxicologically significant enhancer regions, infers that miRNAs are also likely to be of crucial importance in cellular responses to xenobiotics. Indeed, miRNA expression in Drosophila and C. elegans can be regulated by enhancers or hormones (Brennecke et al., 2003; Johnson et al., 2003; Sempere et al., 2003), and various chemotherapeutics and chemicals such as ethanol can control the expression of miRNAs in rodents and humans (Meng et al., 2006; Saito et al., 2006; Pogribny et al., 2007; Rossi et al., 2007; Sathyan et al., 2007). More recently, xenobiotic-mediated miRNA expression has been directly linked with downstream protein expression and cell proliferation in mice (Shah et al., 2007). The peroxisome proliferator-activated receptor alpha $(\operatorname{PPAR} \alpha)$ agonist $\mathrm{Wy}-14,643$ down-regulates the expression of let-7C, which in turn reduces let-7C-mediated repression of c-myc translation. This increases c-mycinduced expression of the proto-oncogenic miR-17-92 cluster, resulting in hepatocyte proliferation (Shah et al., 2007).

There is also evidence for epigenetic modulation of miRNA expression and for miRNAmediated epigenetic alterations. Both DNA methylation and histone modification can affect the expression of a number of miRNAs, and aberrant DNA hypermethylation of miRNAs is being increasingly identified in cancer cells (Saito et al., 2006; Brueckner et al., 2007; Lujambio and Esteller, 2007; Lujambio et al., 2007; Meng et al., 2007; Weber 
et al., 2007). Futhermore, miRNAs themselves appear to play a role in epigenetic modification. The miRNA-29 family reverses aberrant methylation in lung cancer through a direct reduction of DNA methyltransferase (DNMT) 3A and 3B mRNA levels, which leads to decreased expression of the DNMT proteins, reduced global DNA methylation, increased expression of tumour suppressor genes and inhibition of tumour cell growth (Fabbri et al., 2007). In addition, miRNA-140 directly represses histone deacetylase 4 expression (Tuddenham et al., 2006) and may thus regulate chromatin structure. The potential of miRNAs to influence epigenetic changes is supported by the fact that the closely related small interfering RNAs (siRNAs) are also involved in DNA methylation and histone modifications (Chuang and Jones, 2007).

Many miRNA sequences occur within the introns of protein coding genes (Chuang and Jones, 2007; Saetrom et al., 2007). Biosynthesis of such intronic miRNAs relies on pol II transcription and correct splicing of their host genes. This implies that intronic miRNAs are co-regulated with their host genes (Chuang and Jones, 2007) and can thus be under the control of similar enhancer and epigenetic regions as their host genes and nonintronic miRNAs. Furthermore, co-regulation with a miRNA may provide a simple mechanism for a gene to down regulate other genes (Saetrom et al., 2007). It also remains possible that intronic miRNAs have their own promoters and the discovery of $\mathrm{CpG}$ islands within introns suggests that even intronic miRNAs with their own promoters could still be regulated by DNA methylation (Chuang and Jones, 2007). 
Finally, small regulatory RNA molecules, including miRNAs, siRNAs and a recently discovered group of germline-specific PIWI-interacting RNAs (piRNAs) vital for spermatogensis, have been implicated in the inheritance of epigenetic information. In paramutation there is cross-talk between alleles at a single locus, where one allele effectively orders the other to act differently in subsequent generations (Chandler, 2007). One allele appears to act as the 'paramutator' and induces the paramutation (silencing) of the susceptible allele (Ashe and Whitelaw, 2006). The newly paramutated allele can then act as a paramutator on other susceptible alleles and so the phenotype of the paramutator allele is passed down through generations without inheritance of the paramutator allele itself, the offspring remaining genotypically homozygous for the susceptible allele (Ashe and Whitelaw, 2006; Chandler, 2007). This phenomenon, well documented in plants, has recently been reported to occur at a paramutagenic Kit locus in mice (Rassoulzadegan et al., 2006). The same study further revealed that RNA molecules were present in the murine sperm cells and that microinjection of these RNA molecules, or of microRNAs known to target Kit mRNA, into one cell embryos induced the paramutated phenotype. It is therefore possible that the transfer of RNAs such as siRNAs, miRNAs and piRNAs from a sperm to a fertilised egg could play a role in the inheritance of epigenetic modifications such as paramutations (Ashe and Whitelaw, 2006; Rassoulzadegan et al., 2006; Chandler, 2007). Such observations may provide insights into potential mechanisms of transgenerational toxicology.

microRNA target prediction 
miRNAs represent only $0.5-3 \%$ of the genome, but have been predicted to regulate approximately $20-30 \%$ of all human genes (Carthew, 2005). The bioinformatic prediction of animal miRNA targets is complex because they display only modest complementarity to their targets (Du and Zamore, 2005). Various computer programmes are now available for miRNA target prediction and five of the most widely used are reviewed and compared by Sethupathy et al., 2006. Such bioinformatic approaches have suggested that each miRNA has an average of around 200 mRNA targets (Carthew, 2005), however these have to be verified experimentally. To-date very few miRNA-mRNA interactions have been confirmed in the laboratory, and the processes of identifying those worth investigating further can appear very daunting. One way of refining the list of putative targets is to compare the expression patterns of miRNAs with actual changes in mRNA translation.

microRNA expression profiling and global translational change analysis The Systems Toxicology group at the MRC Toxicology Unit has developed methods for profiling miRNA expression and measuring global translational changes using microarray platforms. In order to measure miRNA expression using microarrays some technical challenges must be overcome. Since mature miRNAs are such short molecules, conventional labelling methods are unsuitable. Optimum results have been obtained with the two-stage flashtag ${ }^{\mathrm{TM}}$ kits (Genisphere) that add a poly(A) tail to the miRNA before ligating a fluorophore to the resulting tail (Fig. 2a). The small size and often closely related sequences of miRNAs also provides sensitivity and discrimination problems for traditional DNA-based arrays. The miRCURY ${ }^{\mathrm{TM}}$ LNA probe set (Exiqon) is produced 
from locked nucleic acids (LNAs), which have a locked ribose ring making them high affinity RNA mimics (Castoldi et al., 2006). This means that miRNA expression profiles can be generated with high sensitivity and accuracy from as little as $1 \mu \mathrm{g}$ of total RNA (Castoldi et al., 2006). No miRNA size selection or amplification is required and the microarrays can efficiently distinguish between closely related miRNA family members that may differ by just a single nucleotide (Castoldi et al., 2006).

In a typical microarray experiment, the control RNA sample is labelled with a green fluorophore and the test RNA sample with a red fluorophore. By hybridising both the control and test RNA samples on the same array, any up-regulated miRNAs will be observed as red spots and any down-regulated miRNAs as green spots (Fig. 2b). The $\log _{2}[$ test/control] represents the degree of change. Positive values signify increased miRNA expression in the test sample and negative values to reduced miRNA expression. Verification by both northern blotting using LNA probes (Exiqon) and real-time PCR with kits specifically designed for miRNA detection (Ambion) can be used to confirm microarray results (Fig. 2c-d).

The identification of global translational changes in tissues combines RNA fractionation with expression microarrays. Actively translated mRNAs will have large numbers of ribosomes attached to them, are thus termed polysomes, and are heavier than non-actively translated mRNAs with none or very few ribosomes attached to them (monosomes) (Fig. 3a). This difference in mass allows separation of the two RNA populations by sucrose density centrifugation (Fig. 3a). Real-time PCR using primers for an actively translated 
gene ( $\beta$-actin) and a non-actively translated gene (ATF4) identifies polysomes and monosomes, respectively, confirming successful separation and allowing appropriate pooling of RNA fractions (Fig. 3b). These two pools of mRNA species can be reverse transcribed, labelled and analysed using a suitable probe set, for example the MEEBO probe set (Stanford University and UCSF, sold by Invitrogen).

As with the miRNA arrays, the control RNA sample is labelled with a green fluorophore and the test RNA sample with a red fluorophore. With this type of microarray experiment it is not only changes between the control and test samples that are interesting but also differences between monosomal and polysomal RNA. The latter differences isolate genes that are being actively translated from those being actively transcribed only. Subsequent comparison of the control and test translational changes indicates which genes are being differentially translated within the specific model. Complex mathematical analysis of such an assay can be overcome with a simple experimental design. Raw microarray data is normalised using the fundamental assumption that most of the genes will not be differentially regulated. It is difficult to envisage that this would hold true if monosomal RNA was hybridised against polysomal RNA on the same array. However, the assumption that there will be very little global change between control and test monosomes and between control and test polysomes is much more reasonable and enables appropriate normalisation. Therefore, control monosomal RNA can be hybridised against test monosomal RNA on one array, and control polysomal RNA against test polysomal RNA on a second array. Since the difference between $\log _{2}$ [test polysomes/control polysomes] and $\log _{2}$ [test polysomes/test monosomes] is 
mathematically the same as the difference between $\log _{2}[$ test monosomes/control monosomes] and $\log _{2}$ [control polysomes/control monosomes] then translational changes between the test and control can be identified by comparing the polysome and monosome arrays in this manner (Personal communication, SD Zhang).

Combining the miRNA expression data with the translational data will help to identify potential miRNA-mRNA interactions. Once identified, putative interactions can be further investigated in vitro. A luciferase reporter gene containing the 3'UTR region of the potential mRNA target can be transfected into cells along with the miRNA of interest (Shah et al., 2007). Subsequent inverse correlation of luciferase activity with miRNA expression signifies that the miRNA is binding to its target sequence and either repressing the translation of luciferase mRNA or inducing its degradation. Western blotting can then provide conclusive proof that the validated miRNA-mRNA interaction results in a reduced level of protein and indicates a biological role for the miRNA of interest (Fabbri et al., 2007). Specific miRNAs can also be knocked-down with antisense technology to elucidate potential biological functions (Ji et al., 2007). In the near future, such research will give new insight into the mechanisms by which cells alter their translational profiles in response to toxicity, and will highlight the fundamental roles of miRNA species in the control of such altered translational events in toxicology. Furthermore, since specific miRNAs have already been linked with toxicological processes such as tumour formation, classification and prognosis (Lu et al., 2005; Jay, et al., 2007; Rane, et al., 2007), miRNAs and/or miRNA profiles are also likely to represent important toxicological biomarkers and novel drug targets. 
The future

miRNA field is still in its infancy, however progress is occurring at an extraordinary rate and vast numbers of publications are being generated. Within the space of a few years the science has moved from a naïve unawareness of these small regulatory molecules to a fairly comprehensive understanding of miRNA biosynthesis and the role of miRNA in inhibiting translation. As we uncover more roles for miRNAs in biological processes such as cellular toxicity, disease and epigenetics, and gain greater knowledge of miRNA regulation we discover the next set of challenges. Can we develop better systems for target prediction? Will such systems enable us to identify important regulatory networks in toxicology? How is miRNA expression fully regulated by xenobiotics? Can miRNAs form interlinked regulatory networks with other small regulatory molecules such as siRNAs or piRNAs that are important in developmental and transgenerational toxicology? Do different populations exhibit differential miRNA expression profiles and can this affect susceptibility or resistance to xenobiotic toxicity or disease? What is the global role of miRNAs in cellular toxicity and disease, and do miRNAs provide novel therapeutic opportunities? With such interesting challenges ahead the next few years of miRNA research promise to be very exciting.

\section{Acknowledgements}

The authors would like to thank all members of the Systems Toxicology group at the MRC Toxicology Unit and the laboratory of A Willis and M Bushell for their work and stimulating thought. 


\section{References}

Brennecke, J., Hipfner, D., Stark, A., Russell, R., Cohen, S., 2003. bantam encodes a developmentally regulated microRNA that controls cell proliferation and regulates the proapoptotic gene hid in Drosophila. Cell. 113, 25-36.

Brueckner, B., Stresemann, C., Kuner, R., Mund, C., Musch, T., Meister, M., Sültmann, H., Lyko, F., 2007. The Human let-7a-3 Locus Contains an Epigenetically Regulated MicroRNA Gene with Oncogenic Function. Cancer Res. 67, 1419-1423.

Castoldi, M., Schmidt, S., Benes, V., Noerholm, M., Kulozik, A.E., Hentze, M.W., Muckenthaler, M.U., 2006. A sensitive array for microRNA expression profiling (miChip) based on locked nucleic acids (LNA). RNA. 12, 913-920.

Chuang, J.C., Jones, P.A., 2007. Epigenetics and MicroRNAs. Pediatr. Res. 61, 24R-29R. Du, T., Zamore, P.D., 2005. microPrimer: the biogenesis and function of microRNA. Development. 132, 4645-4652.

Fabbri, M., Garzon, G., Cimmino, A., Liu, Z., Zanesi, N., Callegari, E., Liu, S., Alder, H., Costinean, S., Fernandez-Cymering, C., Volinia, S., Guler, G., Morrison, C.D., Chan, K.K., Marcucci, G., Calin, G.A., Huebner, K., Croce, C.M., 2007. MicroRNA-29 family reverts aberrant methylation in lung cancer by targeting DNA methyltransferases $3 \mathrm{~A}$ and 3B. Proc. Natl. Acad. Sci. USA. 104, 15805-15810. 
Jay, C., Nemunaitis, J., Chen, P., Fulgham, P., Tong, AW., 2007. miRNA profiling for diagnosis and prognosis of human cancer. DNA Cell Biol. 26, 293-300.

Ji, R., Cheng, Y., Yue, J., Yang, J., Liu, X., Chen, H., Dean, D.B., Zhang, C., 2007. MicroRNA expression signature and anti-sense-mediated depletion reveal an essential role of microRNA in vascular neointimal lesion formation. Circ. Res. 100,1579-1588.

Johnson, S., Lin, S., Slack, F., 2003. The time of appearance of the C. elegans let-7 microRNA is transcriptionally controlled utilizing a temporal regulatory element in its promoter. Dev. Biol. 259, 364-379.

Kosik, K.S., 2006. The neuronal microRNA system. Nat. Rev. Neurosci. 7, 911-920.

Lu, J., Getz, G., Miska, E.A., Alvarez-Saavedra, E., Lamb, J., Peck, D., Sweet-Cordero, A., Ebert, B.L., Mak, R.H., Ferrando, A.A., Downing, J.R., Jacks, T., Horvitz, H.R., Golub, T.R., 2005. MicroRNA expression profiles classify human cancers. Nature. 435, 834-838.

Lujambio, A., Esteller, M., 2007. CpG island hypermethylation of tumour suppressor microRNAs in human cancer. Cell Cycle. 6, 1455-1459. 
Lujambio, A., Ropero, S., Ballestar, E., Fraga, M.F., Cerrato, C., Setién, F., Casado, S., Suarez-Gauthier, A., Sanchez-Cespedes, M., Gitt, A., Spiteri, I., Das, P.P., Caldas, C., Miska, E., Esteller, M., 2007. Genetic Unmasking of an Epigenetically Silenced microRNA in Human Cancer Cells. Cancer Res. 67, 1424-1429.

Meister, G., 2007. miRNAs Get an Early Start on Translational Silencing. Cell. 131, 2528.

Meng, F., Henson, R., Lang, M., Wehbe, H., Maheshwari, S., Mendell, J.T., Jiang, J., Schmittgen, T.D., Patel, T., 2006. Involvement of human micro-RNA in growth and response to chemotherapy in human cholangiocarcinoma cell lines. Gastroenterology. 130, 2113-2129.

Meng, F., Wehbe-Janek, H., Henson, R., Smith, H., Patel, T., 2007. Epigenetic regulation of microRNA-370 by interleukin-6 in malignant human cholangiocytes. Oncogene. doi:10.1038/sj.onc. 1210648.

Pogribny, I.P., Tryndyak, V.P., Boyko, A., Rodriguez-Juarez, R., Beland, F.A., Kovalchuk, O., 2007. Induction of microRNAome deregulation in rat liver by long-term tamoxifen exposure. Mutat. Res. 619, 30-37.

Rana, T.M., 2007. Illuminating the silence: understanding the structure and function of small RNAs. Nat. Rev. Mol. Cell Biol. 8, 23-36. 
Rane, S., Sayed, D., Abdellatif, M., 2007. MicroRNA with a MacroFunction. Cell Cycle. $6,1850-1855$.

Rossi, L., Bonmassar, E., Faraoni, I., 2007. Modification of miR gene expression pattern in human colon cancer cells following exposure to 5-flurouracil in vitro. Pharmacol. Res. $56,248-253$.

Saito, Y., Liang, G., Egger, G., Friedman, J.M., Chuang, J.C., Coetzee, G.A., Jones, P.A., 2006. Specific activation of micro-RNA-127 with downregulation of the proto-oncogene BCL6 by chromatin-modifying drugs in human cancer cells. Cancer Cell. 9, 435-443.

Sathyan, P., Golden, H.B., Miranda, R.C., 2007. Competing interactions between microRNAs determine neural progenitor survival and proliferation after ethanol exposure: evidence from an ex vivo model of the fetal cerebral cortical neuroepithelium. J. Neurosci. 27, 8546-8557.

Sempere, L., Sokol, N., Dubrovsky, E., Berger, E., Ambros, V., 2003. Temporal regulation of microRNA expression in Drosophila melanogaster mediated by hormonal signals and broad-Complex gene activity. Dev. Biol. 259, 9-18. 
Sethupathy, P., Megraw, M., Hatzigeorgiou, A., 2006. A guide through present computational approaches for the identification of mammalian microRNA targets. Nat. Methods. 3, 881-886.

Shah, Y.M., Morimura, K., Yang, Q., Tanabe, T., Takagi, M., Gonzalez, F.J., 2007. Peroxisome proliferator-activated receptor alpha regulates a microRNA-mediated signaling cascade responsible for hepatocellular proliferation. Mol. Cell Biol. 27, 42374247.

Shivdasani, R.A., 2006. MicroRNAs:regulators of gene expression and cell differentiation. Blood. 108, 3646-3653.

Tuddenham, L., Wheeler, G., Ntounia-Fousara, S., Waters, J., Hajihosseini, M.K., Clark, I., Dalmay, T., 2006. The cartilage specific microRNA-140 targets histone deacetylase 4 in mouse cells. FEBS Lett. 580, 4214-4217.

Weber, B., Stresemann, C., Brueckner, B., Lyko, F., 2007. Methylation of human microRNA genes in normal and neoplastic cells. Cell Cycle. 6, 1001-1005.

Zhou, X., Ruan, R., Wang, G., Zhang, W., 2007. Characterization and Identification of MicroRNA Core Promoters in Four Model Species. PLoS Comput. Biol. doi:10.1371/journal.pcbi.0030037. 
Figure Legends

Figure 1 miRNA biosysnthesis and function. miRNAs are transcribed as long pri-miRNA transcripts which undergo two processing steps to produce the mature miRNA duplex. The guide strand is incorporated into the RISC complex where it binds to, and inhibits the translation of, its target mRNA.

Figure 2 (a) illustration of flashtag ${ }^{\mathrm{TM}}$ method for labelling of miRNAs (Exiqon), (b) a typical miRNA expression array with changes highlighted in white circles, (c) verification of an up-regulation using Northern blotting and (d) verification of an upregulation using real-time PCR.

Figure 3 (a) representation of the use of RNA fractionation to separate monosomes and polysomes, and (b) verification of successful monosomal and polysomal separation, and identification of RNA fractions for pooling using real-time PCR with markers for polysomes ( $\beta$-actin) and monosomes (ATF4). 


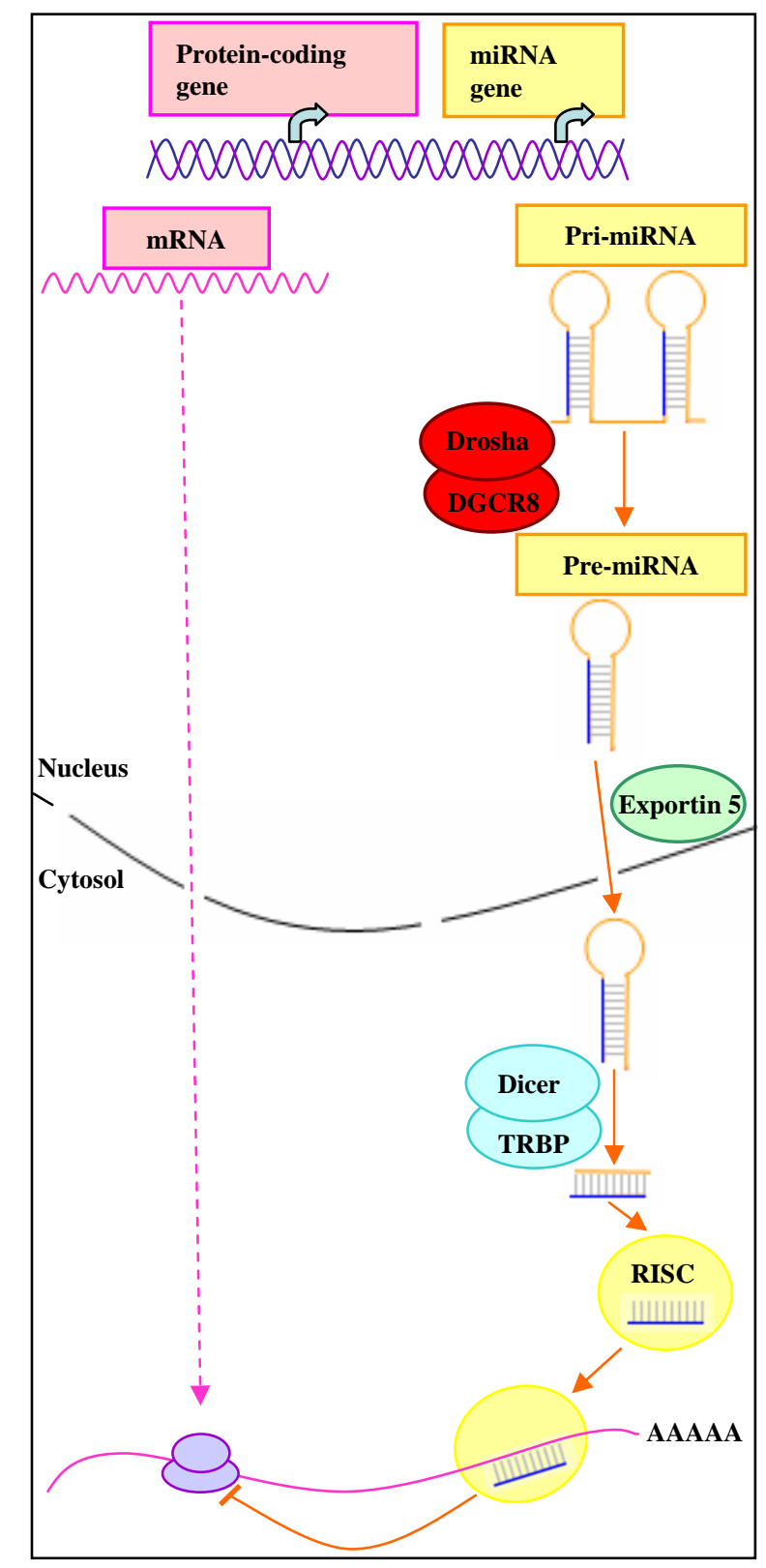




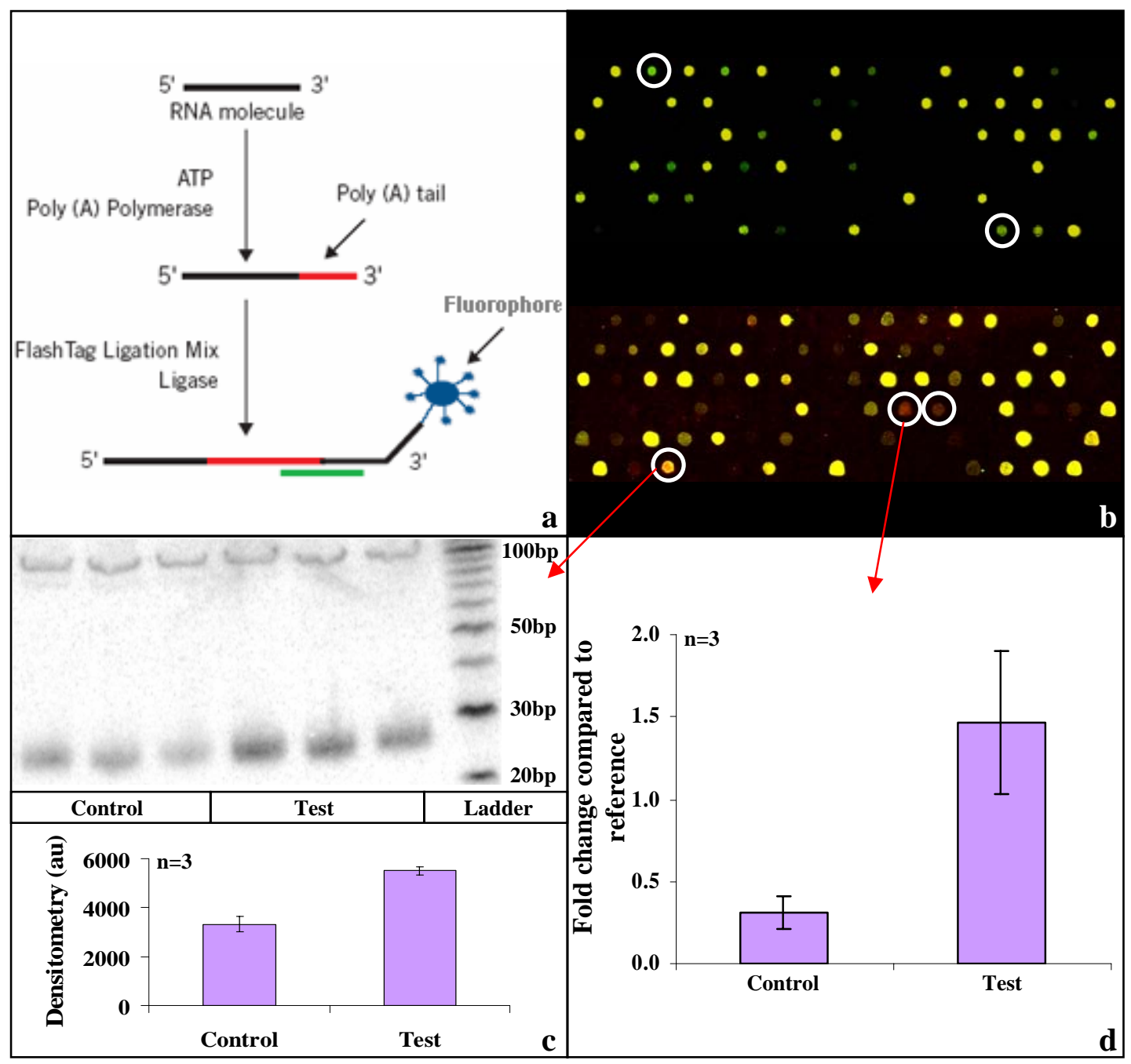




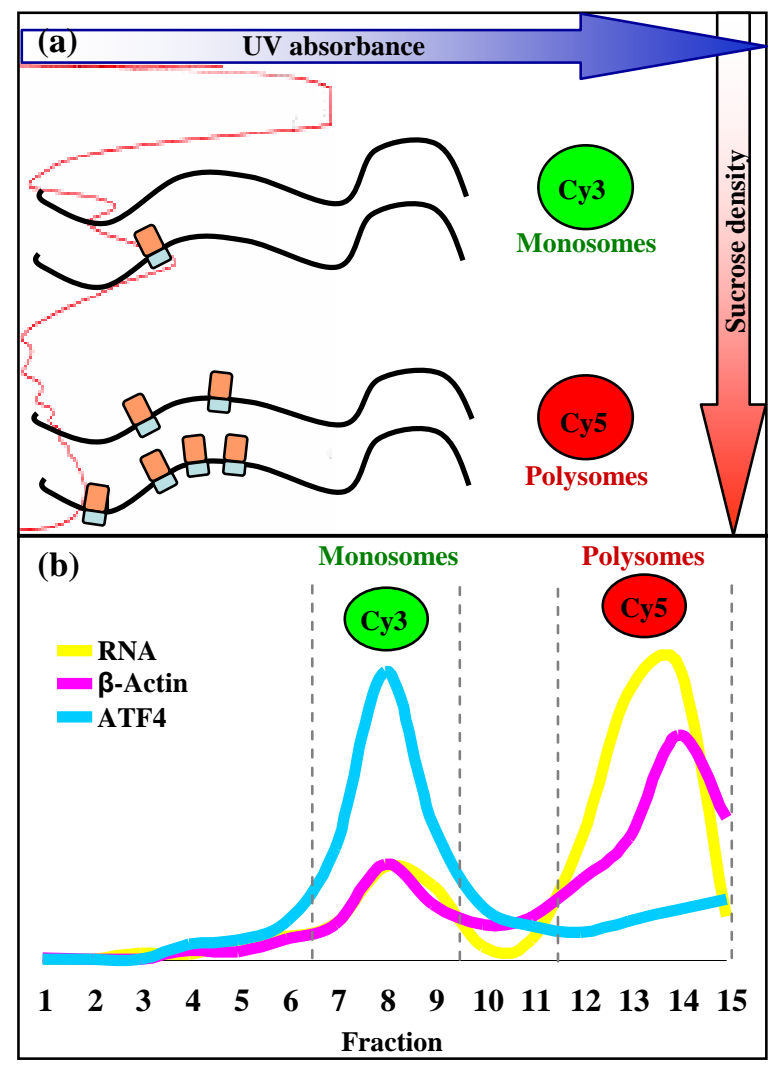

